\title{
LIPSCHITZ EQUIVALENCE OF SUBSETS OF SELF-CONFORMAL SETS
}

\author{
MARTA LLORENTE AND PERTTI MATTILA
}

\begin{abstract}
We give sufficient conditions to guarantee that if two self-conformal sets $E$ and $F$ have Lipschitz equivalent subsets of positive measure, then there is a bilipschitz map of $E$ into, or onto, $F$.
\end{abstract}

\section{INTRODUCTION}

In this note we shall consider the following question: suppose that $E$ and $F$ are self-conformal (see below for terminology) subsets of $\mathbb{R}^{n}$ of the same Hausdorff dimension $s$. If there are measurable subsets $E^{\prime} \subset E$ and $F^{\prime} \subset F$ of positive $s$-dimensional Hausdorff measure which are Lipschitz equivalent, are then also $E$ and $F$ Lipschitz equivalent? By Lipschitz equivalence we mean that there is a bilipschitz map of $E$ onto $F$. We shall prove that this is true for some Cantor type sets, more precisely, when $E$ and $F$ satisfy the strong separation condition and one of these sets is generated by two maps. If $E$ and $F$ satisfy the open set condition we shall show that there is a bilipschitz map of $E$ into $F$, but not necessarily onto.

Lipschitz equivalence of self-similar and self-conformal sets has been considered in [FM], [RRX] and [RRY], and a general study of sets having many Lipschitz equivalent subsets can be found in [DS]. In particular, Falconer and Marsh gave necessary algebraic conditions on the similarity ratios of the generating maps in order that two sets satisfying strong separation condition could be Lipschitz equivalent. These imply, for example, that many self similar subsets of Hausdorff dimension $\log 2 / \log 3$ are not Lipschitz equivalent with the classical 1/3-Cantor set. Hence by our result in such a case neither are any of their subsets of positive measure. In $\mathrm{MS}$ ] it was shown for the much larger

2000 Mathematics Subject Classification. Primary 28A75, 28A80.

Key words and phrases. Bilipschitz maps, self-similar sets, self-conformal sets.

Part of this job has been done while MLL was visiting the University of Helsinki and supported by the Academy of Finland. MLL was also supported by the Ministerio de Educacion y Ciencia, research project MTM2006-02372.

PM was supported by the Academy of Finland. 
class of Ahlfors-David regular sets $E \subset \mathbb{R}^{n}$ and $F \subset \mathbb{R}^{n}$ of dimensions $s<t<1$, respectively, that $E$ is Lipschitz equivalent to some subset of $F$. Combining the results of Falconer and Marsh and the results of this paper we see that this cannot be extended to the case $s=t$.

\section{Preliminaries}

We shall denote the closed ball with center $x$ and radius $r$ by $B(x, r)$. The diameter of a set $A$ is denoted by $d(A)$. A map $h: A \rightarrow B, A \subset$ $\mathbb{R}^{n}, B \subset \mathbb{R}^{p}$, is said to be bilipschitz, or $L$-bilipschitz, if there is $L<\infty$ such that

$$
|x-y| / L \leq|h(x)-h(y)| \leq L|x-y| \text { for all } x, y \in A .
$$

The smallest such a constant $L$ is denoted by $\operatorname{bilip}(h)$. Note that we don't require $h$ to be onto.

We shall make use of the following simple lemma:

2.1. Lemma. Let $A_{k} \subset A \subset \mathbb{R}^{n}, B_{k} \subset B \subset \mathbb{R}^{p}$, be compact and $h_{k}: A_{k} \rightarrow B_{k}, k=1,2, \ldots$, be such that for some $L, 0<L<\infty$, $|x-y| / L \leq\left|h_{k}(x)-h_{k}(y)\right| \leq L|x-y|$ for all $x, y \in A_{k}, k=1,2, \ldots$

If for every $x \in A$ there are $x_{k} \in A_{k}$ with $x_{k} \rightarrow x$, then there is an L-bilipschitz map $h: A \rightarrow B$. If also $h_{k}\left(A_{k}\right)=B_{k}$ and for every $y \in B$ there are $y_{k} \in B_{k}$ with $y_{k} \rightarrow y$, then $h(A)=B$.

Proof. We can extend the maps $h_{k}$ to $L$-Lipschitz maps $\mathbb{R}^{n} \rightarrow \mathbb{R}^{p}$; we shall denote by $h_{k}$ also the extended maps. By the Arzela-Ascoli theorem the sequence $\left(h_{k}\right)$ has a subsequence which converges uniformly on compact subsets of $\mathbb{R}^{n}$ to an $L$-Lipschitz map $h$. It is easy to check that $h \mid A: A \rightarrow B$ is $L$-bilipschitz, and also the last claim is simple.

We denote by $\mathcal{H}^{s}$ the $s$-dimensional Hausdorff measure. We shall use the fact (see, e.g. Theorem 6.2 in [M]) that for any $\mathcal{H}^{s}$ measurable sets $A \subset E \subset \mathbb{R}^{n}$ with $\mathcal{H}^{s}(E)<\infty, \mathcal{H}^{s}$ almost all points $x \in A$ are density points of $A$ with respect to $E$ in the sense that

$$
\lim _{r \rightarrow 0} r^{-s} \mathcal{H}^{s}(B(x, r) \cap E \backslash A)=0 .
$$

We shall consider a conformal iterated function system $\left\{f_{1}, \ldots, f_{N}\right\}$ in $\mathbb{R}^{n}$ following the scheme of [MU]. By this we mean that $N \geq 2$ and there is an open connected set $V \subset \mathbb{R}^{n}$ such that each $f_{i}: V \rightarrow V$ is an injective conformal contraction;

$$
\left|f_{i}(x)-f_{i}(y)\right| \leq L_{0}<1 \text { for all } x, y \in V, i=1, \ldots, N,
$$

of class $C^{1+\gamma}$ for some fixed $\gamma>0$, that is, the partial derivatives are Hölder continuous with exponent $\gamma$. Of course, the last condition is only 
needed when $n=1$, since the conformal maps in higher dimensions are $C^{\infty}$. We shall also assume that there are positive constants $c_{1}$ and $C_{1}$ such that $0<c_{1}<C_{1}<1$ and

$$
c_{1} \leq\left\|D f_{i}(x)\right\| \leq C_{1} \text { for all } x \in V, i=1, \ldots, N .
$$

Here $\|\cdot\|$ is the operator norm of a linear map. Then there is a unique compact invariant set $E$ such that (see $[\mathrm{H}]$ )

$$
E=\bigcup_{i=1}^{N} f_{i}(E) .
$$

We shall use the following notation. Let $\mathcal{N}=\{1,2, \ldots, n\}$ and

$$
\mathcal{N}^{k}=\left\{\mathbf{i}=\left(i_{1}, \ldots, i_{k}\right): i_{j} \in \mathcal{N} \forall j=1, \ldots k\right\} .
$$

For $\mathbf{i}=\left(i_{1}, \ldots, i_{k}\right) \in \mathcal{N}^{k}$ let

$$
\begin{gathered}
f_{\mathbf{i}}=f_{i_{1}} \circ f_{i_{2}} \circ \ldots \circ f_{i_{k}}, \\
E_{\mathbf{i}}=f_{\mathbf{i}}(E), \\
d_{\mathbf{i}}=d\left(E_{\mathbf{i}}\right) .
\end{gathered}
$$

Then for every $k=1,2, \ldots$,

$$
E=\bigcup_{\mathbf{i} \in \mathcal{N}^{k}} f_{\mathbf{i}}(E)
$$

We shall assume that the system $\left\{f_{i}\right\}$ satisfies the open set condition, that is, there is a non-empty bounded open set $O \subset V$ such that the closure of $O$ is contained in $V$ and the sets $f_{i}(O)$ are disjoint subsets of $O$. Then the following bounded distortion property holds, see [MU], Remark 2.3: there is constant $K$ such that for $\mathbf{i} \in \mathcal{N}^{k}$,

$$
\left\|D f_{\mathbf{i}}(x)\right\| \leq K\left\|D f_{\mathbf{i}}(y)\right\| \text { for all } x, y \in V \text {. }
$$

Let $s$ be the Hausdorff dimension of $E$. The open set condition implies (and it is in fact equivalent to) that $0<\mathcal{H}^{s}(E)<\infty$, see [PRSS]. From the bounded distortion property one can conclude that there exist positive constants $c<1, C$ and $R$ such that for all $\mathbf{i}=\left(i_{1}, \ldots, i_{k}\right) \in \mathcal{N}^{k}$ and $\mathbf{i} i=\left(i_{1}, \ldots, i_{k}, i\right) \in \mathcal{N}^{k+1}$,

$$
\begin{gathered}
c d_{\mathbf{i}} \leq\left\|D f_{\mathbf{i}}(x)\right\| \leq C d_{\mathbf{i}} \text { for } x \in V \\
B\left(f_{\mathbf{i}}(x), c d_{\mathbf{i}} r\right) \subset f_{\mathbf{i}}(B(x, r)) \text { for } x \in E, 0<r<R \\
c d_{\mathbf{i}}|x-y| \leq\left|f_{\mathbf{i}}(x)-f_{\mathbf{i}}(y)\right| \leq C d_{\mathbf{i}}|x-y| \text { for } x, y \in E \\
\qquad d_{\mathbf{i}} \leq C d_{\mathbf{i} i}, \\
d_{\mathbf{i}} \leq L_{0}^{k} d(E), d_{\mathbf{i}} \rightarrow 0 \text { when } \mathbf{i} \in \mathcal{N}^{k}, k \rightarrow \infty
\end{gathered}
$$




$$
c r^{s} \leq \mathcal{H}^{s}(B(x, r) \cap E) \leq C r^{s} \text { for } x \in E, 0<r<R .
$$

Here (2.4) and (2.5) are proven in Section 2 of [MU, (2.6) follows easily from (2.4), (2.7) follows from (2.6), (2.8) follows from (2.3), and (2.9) is proven in Lemma 3.14 of [MU] (where a measure $m$ is used instead of $\mathcal{H}^{s}$, but this is equivalent).

We shall assume all the time that $f_{\mathbf{i}}, E, E_{\mathbf{i}}, d_{\mathbf{i}}, s, c$ and $C$ are as above. We shall also consider another conformal iterated function system $\left\{g_{1}, \ldots, g_{P}\right\}$ in $\mathbb{R}^{p}$ and use the corresponding notation $g_{\mathbf{i}}, F, F_{\mathbf{i}}, e_{\mathbf{i}}, s, c$ and $C$; in particular we assume that $E$ and $F$ have the same Hausdorff dimension $s$ and we choose the constants $c$ and $C$ so that they match both systems.

\section{Open SET CONDITION}

We shall use the result of Peres, Rams, Simon and Solomyak from [PRSS (proven first by Schief in [S] for self-similar sets) according to which the open set condition is equivalent to the strong open set condition: for some open set $O$ as in the open set condition $E \cap O \neq \emptyset$. Both are also equivalent with $0<\mathcal{H}^{s}(E)<\infty$.

It is easy to see that if $O$ is as in the open set condition, then $E \subset \bar{O}$, and so for all $\mathbf{i} \in \mathcal{N}^{k}, E_{\mathbf{i}} \subset f_{\mathbf{i}}(\bar{O})$. Using the strong open set condition, choose $x_{0} \in E \cap O$ and $r_{0}, 0<r_{0}<1$, such that $B\left(x_{0}, r_{0}\right) \subset O$. Then for all $\mathbf{i} \in \mathcal{N}^{k}$ by (2.5),$B\left(f_{\mathbf{i}}\left(x_{0}\right), c d_{\mathbf{i}} r_{0}\right) \subset f_{\mathbf{i}}\left(B\left(x_{0}, r_{0}\right)\right) \subset f_{\mathbf{i}}(O)$, from which it follows that

$$
\left(E \backslash E_{\mathbf{i}}\right) \cap B\left(f_{\mathbf{i}}\left(x_{0}\right), c d_{\mathbf{i}} r_{0}\right)=\emptyset .
$$

3.2. Lemma. Suppose that the system $\left\{f_{1}, \ldots, f_{N}\right\}$ satisfies the open set condition. Let $b=c r_{0} / 2$ where $r_{0}$ is as above. Then for $\mathcal{H}^{s}$ almost all $x \in E$ there are $\mathbf{i}_{k}, k=1,2, \ldots$, such that $x \in E_{\mathbf{i}_{k}}, d_{\mathbf{i}_{k}} \rightarrow 0$ and $\left(E \backslash E_{\mathbf{i}_{k}}\right) \cap B\left(x, b d_{\mathbf{i}_{k}}\right)=\emptyset$ for all $k=1,2, \ldots$

Proof. Let $A_{m}, m=1,2, \ldots$, be the set of $x \in E$ such that $\left(E \backslash E_{\mathbf{i}}\right) \cap$ $B\left(x, b d_{\mathbf{i}}\right) \neq \emptyset$ whenever $x \in E_{\mathbf{i}}$ and $d_{\mathbf{i}}<1 / m$. We shall show that $\mathcal{H}^{s}\left(A_{m}\right)=0$ which implies the lemma.

Let $x \in A_{m}$. If $x \in E_{\mathbf{i}}$ and $d_{\mathbf{i}}<1 / m$, then by (3.1) and the choice of $b$,

$$
\left(E \backslash E_{\mathbf{i}}\right) \cap B\left(f_{\mathbf{i}}\left(x_{0}\right), 2 b d_{\mathbf{i}}\right)=\emptyset,
$$

and so

$$
\left(E \backslash E_{\mathbf{i}}\right) \cap B\left(y, b d_{\mathbf{i}}\right)=\emptyset \text { for all } y \in B\left(f_{\mathbf{i}}\left(x_{0}\right), b d_{\mathbf{i}}\right),
$$

whence

$$
B\left(f_{\mathbf{i}}\left(x_{0}\right), b d_{\mathbf{i}}\right) \subset B\left(x,(1+b) d_{\mathbf{i}}\right) \backslash A_{m} .
$$


Therefore by (2.9),

$$
\mathcal{H}^{s}\left(B\left(x,(1+b) d_{\mathbf{i}}\right) \cap E \backslash A_{m}\right) \geq c\left(b d_{\mathbf{i}}\right)^{s} .
$$

It follows that $x$ cannot be a density point of $A_{m}$ and proves that $\mathcal{H}^{s}\left(A_{m}\right)=0$.

3.3. Theorem. Suppose that the systems $\left\{f_{1}, \ldots, f_{N}\right\}$ and $\left\{g_{1}, \ldots, g_{P}\right\}$ satisfy the open set condition. If there are an $\mathcal{H}^{s}$ measurable subset $E^{\prime}$ of $E$ with $\mathcal{H}^{s}\left(E^{\prime}\right)>0$ and a bilipschitz map $h: E^{\prime} \rightarrow F$, then there exists a bilipschitz map $\tilde{h}: E \rightarrow F$.

Proof. We may assume that $E^{\prime}$ is compact. Let $x \in E^{\prime}$ be a density point of $E^{\prime}$ such that also $y=h(x)$ is a density point of $F^{\prime}=h\left(E^{\prime}\right)$ and that, using Lemma 3.2, there are $b>0$ and $\mathbf{j}_{k}$ such that $y \in F_{\mathbf{j}_{k}}, e_{\mathbf{j}_{k}} \rightarrow 0$ and $\left(F \backslash F_{\mathbf{j}_{k}}\right) \cap B\left(y, b e_{\mathbf{j}_{k}}\right)=\emptyset$ for all $k=1,2, \ldots$ Let $L$ be the bilipschitz constant of $h$. For $k=1,2, \ldots$, let $\mathbf{i}_{k}$ be a multi-index of shortest length such that $x \in E_{\mathbf{i}_{k}}$ and $L d_{\mathbf{i}_{k}} \leq b e_{\mathbf{j}_{k}}$. Then

$$
h\left(E^{\prime} \cap E_{\mathbf{i}_{k}}\right) \subset B\left(y, L d_{\mathbf{i}_{k}}\right) \cap F^{\prime} \subset B\left(y, b e_{\mathbf{j}_{k}}\right) \cap F^{\prime} \cap F_{\mathbf{j}_{k}},
$$

and, by the minimality of $\mathbf{i}_{k}$ and (2.7), if $\mathbf{i}_{k}=\mathbf{i} i$, then

$$
d_{\mathbf{i}_{k}} \geq C^{-1} d_{\mathbf{i}} \geq(C L)^{-1} b e_{\mathbf{j}_{k}} .
$$

Denote

$$
h_{k}=g_{\mathbf{j}_{k}}^{-1} \circ h \circ f_{\mathbf{i}_{k}}: A_{k}:=f_{\mathbf{i}_{k}}^{-1}\left(E^{\prime} \cap E_{\mathbf{i}_{k}}\right) \rightarrow F .
$$

Then by (2.6) $h_{k}$ is bilipschitz with bilip $\left(h_{k}\right) \leq L^{\prime}$ with $L^{\prime}$ independent of $k$. To complete the proof we shall check that the condition of Lemma 2.1 holds for $A_{k}$ and $A=E$.

Suppose it doesn't. Then there are $a \in E$ and $r, 0<r<R$, such that for some subsequence of $\left(\mathbf{i}_{k}\right)$, which we assume to be the full sequence, we have $B(a, r) \cap f_{\mathbf{i}_{k}}^{-1}\left(E^{\prime} \cap E_{\mathbf{i}_{k}}\right)=\emptyset$. Then by (2.5)

$$
B\left(f_{\mathbf{i}_{k}}(a), c d_{\mathbf{i}_{k}} r\right) \cap E_{\mathbf{i}_{k}} \subset f_{\mathbf{i}_{k}}(B(a, r)) \cap E_{\mathbf{i}_{k}} \subset B\left(x, d_{\mathbf{i}_{k}}\right) \cap\left(E \backslash E^{\prime}\right) .
$$

This gives by (2.9) that

$$
\mathcal{H}^{s}\left(B\left(x, d_{\mathbf{i}_{k}}\right) \cap\left(E \backslash E^{\prime}\right)\right) \geq c^{s+1}\left(d_{\mathbf{i}_{k}} r\right)^{s} .
$$

This contradicts the fact that $x$ is a density point and proves the theorem.

3.4. Remark. We would like to thank Tamas Keleti for the following observation: in Theorem 3.3 we cannot always get the map $\tilde{h}$ to be onto. To see this, take in Theorem $3.3 E^{\prime}=E \subset \mathbb{R}$ to be a self-similar set satisfying the open set condition with positive Lebesgue measure and $E$ not being an interval, $F$ a compact interval containing $E$ and 
$h=i d$, the identity map. That such a set $E$ exists can be seen for example from $[\mathrm{B}]$.

Also note that, as a straightforward consequence of Theorem 3.3 , we find that a self-similar set in $\mathbb{R}^{n}$ with open set condition and positive Lebesgue measure must have a non-empty interior. A simple direct proof for this fact can be found in [S].

\section{Strong Separation CONDition}

We say that the strong separation condition holds if the sets $f_{i}(E), i=$ $1, \ldots, N$, are disjoint. Then we can choose the constant $c$ so that, in addition to the previous properties,

$$
\operatorname{dist}\left(E_{\mathbf{i} i}, E_{\mathbf{i} j}\right) \geq c d_{\mathbf{i}} \text { for } i \neq j .
$$

4.2. Theorem. Suppose that $p=2$ and the systems $\left\{f_{1}, \ldots, f_{N}\right\}$ and $\left\{g_{1}, g_{2}\right\}$ satisfy the strong separation condition. If there are an $\mathcal{H}^{s}$ measurable subset $E^{\prime}$ of $E$ with $\mathcal{H}^{s}\left(E^{\prime}\right)>0$ and a bilipschitz map $h: E^{\prime} \rightarrow F$, then there exists a bilipschitz map $\tilde{h}: E \rightarrow F$ with $\tilde{h}(E)=F$.

Proof. By Theorem 3.3, we may assume that $E^{\prime}=E$. Let $x \in E$ be such that $y=h(x)$ is a density point of $F^{\prime}=h(E)$. For every $k \in \mathbb{N}$, choose $E_{\mathbf{i}_{k}}$ such that $x \in E_{\mathbf{i}_{k}}$ and $d_{\mathbf{i}_{k}} \rightarrow 0$. Then there are sets $F_{k, l}=F_{\mathbf{j}_{k(l)}}$ and the corresponding maps $g_{k, l}=g_{\mathbf{j}_{k(l)}}, l=1, \ldots m_{k}$, such that $d\left(F_{k, l}\right) \geq c_{1} d_{\mathbf{i}_{k}}$ and $m_{k} \leq m$, where $c_{1}>0$ and $m$ are independent of $k, F^{\prime} \cap F_{k, l} \neq \emptyset$ and

$$
h\left(E_{\mathbf{i}_{k}}\right)=\bigcup_{l=1}^{m_{k}} F^{\prime} \cap F_{k, l} .
$$

This is essentially Lemma 3.2 in [FM] but we give a quick proof. By (4.1) $\operatorname{dist}\left(E_{\mathbf{i}_{k}}, E_{\lambda}\right) \geq c d_{\mathbf{i}_{k}}$ whenever $E_{\mathbf{i}_{k}} \cap E_{\lambda}=\emptyset$. If for such $\lambda$ and for some $\mathbf{j}, h\left(E_{\mathbf{i}_{k}}\right) \cap F_{\mathbf{j}} \neq \emptyset$ and $h\left(E_{\lambda}\right) \cap F_{\mathbf{j}} \neq \emptyset$, then $d\left(F_{\mathbf{j}}\right) \geq$ $\operatorname{dist}\left(h\left(E_{\mathbf{i}_{k}}\right), h\left(E_{\lambda}\right)\right) \geq(c / L) d_{\mathbf{i}_{k}}$ where $L$ is the bilipschitz constant of $h$. Therefore we can take as $F_{k, l}$ all the maximal sets $F_{\mathbf{j}}$ such that $h\left(E_{\mathbf{i}_{k}}\right) \cap F_{\mathbf{j}} \neq \emptyset$ and $d\left(F_{\mathbf{j}}\right)<(c / L) d_{\mathbf{i}_{k}}$. Denoting $d_{k, l}=d\left(F_{k, l}\right)$ we have then by (2.7) $($ as $c / L \leq 1)$,

$$
c(L C)^{-1} d_{\mathbf{i}_{k}} \leq d_{k, l} \leq d_{\mathbf{i}_{k}} .
$$

Since $p=2$, we can choose disjoint sets $\tilde{F}_{k, i}=F_{\mathbf{j}_{l(i)}}, i=1, \ldots, m_{k}$, such that $d\left(\tilde{F}_{k, i}\right) \geq c_{2}$, with $c_{2}>0$ independent of $k$, and $F=\cup_{i=1}^{m_{k}} \tilde{F}_{k, i}$. Let $\tilde{g}_{k, i}$ be the corresponding maps. Note that the maps $\tilde{g}_{k, i}$ are selected 
from a fixed finite family, so their bilipschitz constants have an upper bound independent of $k$. Define

$$
h_{k}: E \rightarrow F
$$

by setting

$$
h_{k}(x)=\tilde{g}_{k, i}\left(g_{k, i}^{-1}\left(h\left(f_{\mathbf{i}_{k}}(x)\right)\right)\right) \text { if } x \in f_{\mathbf{i}_{k}}^{-1}\left(h^{-1}\left(F^{\prime} \cap F_{k, i}\right)\right) .
$$

Then $\operatorname{bilip}\left(h_{k}\right) \leq L^{\prime}$ where $L^{\prime}$ is independent of $k$. Namely, the case when $x, y \in f_{\mathbf{i}_{k}}^{-1}\left(h^{-1}\left(F^{\prime} \cap F_{k, i}\right)\right)$, follows by composition. Furthermore, the strong separation condition provides us with constants $M_{1}, M_{2}>0$ such that

and

$$
\operatorname{dist}\left(\tilde{F}_{k, i}, \tilde{F}_{k, j}\right) \geq M_{1}
$$

$$
\operatorname{dist}\left(F_{k, i}, F_{k, j}\right) \geq M_{2} d_{\mathbf{i}_{k}}
$$

for all $i \neq j, i, j=1, \ldots m_{k}$. This takes care of the case $x \in f_{\mathbf{i}_{k}}^{-1}\left(h^{-1}\left(F^{\prime} \cap F_{k, i}\right)\right)$, $y \in f_{\mathbf{i}_{k}}^{-1}\left(h^{-1}\left(F^{\prime} \cap F_{k, j}\right)\right)$ with $i \neq j$.

We still need to check that the sets

$$
h_{k}(E)=\bigcup_{i=1}^{m_{k}} \tilde{g}_{k, i}\left(g_{k, i}^{-1}\left(F^{\prime} \cap F_{k, i}\right)\right)
$$

and $F$ satisfy the condition for $B_{k}$ and $B$ in Lemma 2.1. Suppose this is not so. Then there are $a \in F$ and $r, 0<r<R$, such that $B(a, r) \cap h_{k}(E)=\emptyset$ for some arbitrarily large $k$. For such a $k, a$ belongs to some $\tilde{F}_{k, i_{0}}$. For some $c_{3}>0$ independent of $k, B\left(\tilde{g}_{k, i_{0}}^{-1}(a), c_{3} r\right) \subset$ $\tilde{g}_{k, i_{0}}^{-1}(B(a, r))$ and so, with $b=g_{k, i_{0}}\left(\tilde{g}_{k, i_{0}}^{-1}(a)\right)$ by (2.5),

$$
B\left(b, c_{3} c r d_{k, i_{0}}\right) \subset g_{k, i_{0}}\left(\tilde{g}_{k, i_{0}}^{-1}(B(a, r))\right) \subset \mathbb{R}^{n} \backslash F^{\prime} .
$$

Since $y \in h\left(E_{\mathbf{i}_{k}}\right), b \in F_{k, i_{0}}, F_{k, i_{0}} \cap h\left(E_{\mathbf{i}_{k}}\right) \neq \emptyset$, and $d_{k, i_{0}} \leq d_{\mathbf{i}_{k}}$, by (4.3), we have $d(y, b) \leq(L+1) d_{\mathbf{i}_{k}}$ and so by (4.3),

$B\left(b, c_{4} d_{\mathbf{i}_{k}}\right) \subset B\left(b, c_{3} c r d_{k, i_{0}}\right) \subset B\left(y,(L+1) d_{\mathbf{i}_{k}}+c_{3} r d_{k, i_{0}}\right) \subset B\left(y, c_{5} d_{\mathbf{i}_{k}}\right)$

with $c_{4}=c_{3} c^{2}(L C)^{-1} r, c_{5}=L+1+c_{3} r$. Hence by (2.9),

$$
\mathcal{H}^{s}\left(B\left(y, c_{5} d_{\mathbf{i}_{k}}\right) \cap\left(F \backslash F^{\prime}\right)\right) \geq \mathcal{H}^{s}\left(B\left(b, c_{4} d_{\mathbf{i}_{k}}\right) \cap F\right) \geq c\left(c_{4} d_{\mathbf{i}_{k}}\right)^{s}
$$

contradicting the fact that $y$ is a density point of $F^{\prime}$.

4.4. Remark. We don't know if the condition $p=2$ is needed in Theorem 4.2. Clearly the proof gives for general $p$ that there is a bilipschitz map of $E$ onto $F_{0}$ where $F_{0}$ is a finite union of sets $F_{\mathbf{j}}$. This raises a question: under what conditions is such a union Lipschitz equivalent with $F$ ? 
Falconer and Marsh proved in [FM] that for self-similar sets $E$ and $F$ satisfying the strong separation condition the Lipschitz equivalence of $E$ and $F$ implies certain algebraic conditions on the similarity ratios of the generating maps. Possibly their method could be modified to prove the same conditions already if $E$ and $F$ have Lipschitz equivalent measurable subsets of positive measure. However, we could not deduce from this the Lipschitz equivalence of $E$ and $F$ in general since it is not clear when the necessary conditions of Falconer and Marsh are also sufficient.

\section{REFERENCES}

[B] C. BANDT: Self-similar sets 5. Integer matrices and fractal tilings of $\mathbb{R}^{n}$, Proc. Amer. Math. Soc., 112 (1991), 549-562.

[DS] G. David and S. Semmes: Fractured Fractals and Broken Dreams, Oxford University Press, 1997.

[FM] K.J. FALCONER and D.T. MARSh: On the Lipschitz equivalence of Cantor sets, Mathematika, 39 (1992), 223-233.

[H] J.E. Hutchinson: Fractals and self similarity, Indiana Univ. Math. J., 30 (1981), 713-747.

[M] P. Mattila: Geometry of Sets and Measures in Euclidean Spaces, Cambridge University Press, 1995.

[MS] P. Mattila and P. SaAranen: Ahlfors-David regular sets and bilipschitz maps, Ann. Acad. Sci. Fenn., 34 (2009), 487-502.

[MU] R.D. MaUldin and M. URbanski: Dimensions and measures in infinite iterated function systems. Proc. London Math. Soc.(3), 73, no. 1 (1996), 105-154.

[PRSS] Y. Peres, M. Rams, K. Simon and B. Solomyak: Equivalence of positive Hausdorff measure and the open set condition for self-conformal sets, Proc. Amer. Math. Soc 129 (2001), 2689-2699.

[RRX] H. RAO, H.-J. RUAN and L.-F. XI: Lipschitz equivalence and self-similar sets, C. R. Math. Acad. Sci. Paris 342, no. 3 (2006), 191-196.

[RRY] H. RAO, H.-J. RUAN and Y.-M. YANG: Gap sequence, Lipschitz equivalence and box dimension of fractals, Nonlinearity 21 (2008), 1339-1347.

[S] A. Schief: Separation properties for self-similar sets. Proc. Amer. Math. Soc. 122, no. 1 (1994), 111-115.

Marta Llorente: Departamento de Análisis Económico: Economía Cuantitativa, Universidad Autònoma de Madrid, Campus de CantoBLANCO 28049 MADRID, SPAIN,

E-mail address: m.llorente@uam.es

Pertti Mattila: Department of Mathematics and Statistics, Fi00014 University of Helsinki, Finland,

E-mail address: pertti.mattila@helsinki.fi 